\title{
LIFETIME FOR WIRELESS SENSOR NETWORK ANALYSIS OF MIMO COOPERATIVE BY USING DFT
}

\author{
Raed S. M. Daraghma \\ Department of Electrical and Electronics Engineering, Palestine Technical University, Palestine \\ R.daraghmeh@ptuk.edu.ps
}

\begin{abstract}
In this paper, cooperative Multiple Input Multiple Output (MIMO) sensor network based on Discrete Fourier Transform (DFT) in Wireless Sensor Network (WSN) is proposed to improve the network lifetime. The performance of proposed MIMO cooperative DFT scheme is evaluated in terms of energy efficient and reliability. The transmission power is reduced by using MIMO cooperative DFT scheme, Bit Error Rate (BER) analysis for BPSK, QPSK, 16 PSK modulations in MIMO cooperative DFT is presented. Simulation outcomes display that the network lifetime of the suggested MIMO cooperative scheme enhances 1.8 times compared with MIMO cooperative WSN. Outcomes display that when the Accesses Points (AP) is above a certain height, the suggested MIMO cooperative DFT protocol can remarkably extend network lifetime compared with other cooperative protocols.
\end{abstract}

Keyword - Cooperative MIMO, DFT, Lifetime, Wireless Sensor Network, BER

\section{INTRODUCTION}

In [1], the author discussed several operational advantages for Radar sensor network, including bistatic and multi-static radars. In radar sensor networks, each Radar can be an independent sensor that sends a particular waveform and receives the identical returns.

In [2], the author carries out vast numerical simulations with distinct waveforms that can be applied in allocated radar sensor networks, like down-chip and up-chip waveforms, the slightly interfere or opposite chirp rate LFM waveform and the OFDM chirp varied waveform.

In [3], the author developed a new dispensed transmission strategy, which employs the domestic information on both the channel state and the residual energy of sensor nodes. Moreover, he showed that the network lifetime depends on not only the initial energy of the sensors and the number of sensors but also the average transmission energy and the average residual energy in the network.

In [4], the author considered MIMO systems based on Altamonte diversity scheme. By transmitting and receiving information jointly, he showed that through several distance ranges, cooperative MIMO transmission and reception can simultaneously obtain both energy savings and delay decrease.

In [5], several cooperative protocols like amplify and forward (AF), decoded and forward (DF), and space time coded cooperation have been suggested.

In [6], the author proposed a modern divided cooperative MAC protocol to enhance the lifetime of WSN. In this protocol, both channel state information (CSI) and residual energy information (REI) of sensor nodes are counted to select the cooperative nodes.

In [7], the author proposed a new energy active clustering based protocol for single-hop heterogeneous WSN. The proposed protocol employs channel state information in the election procedure of cluster heads $(\mathrm{CHs})$. Cluster heads are chosen in each cluster on the basis of residual node energy and the best channel.

In [8], the author proposed energy saving improvement technique to increase stability period of WSNs, optimal number of clusters are used and cluster heads are selected based on residual energies and distances.

In this work, we present and assesse a new MIMO cooperative energy efficient strategy based on DFT for wireless sensor network. We are particularly interested to extend the lifetime of WSN.

The paper is arranged as follows: in Section II, MIMO cooperative DFT is presented. The performance of MIMO cooperative DFT is assessed through simulations via MATLAB in Section III. Some understanding comments are stated in Section IV.

\section{MIMO COOPERATIVE DFT MODEL}

Let us consider a model as shown in Fig.1, a wireless radar network with $\mathrm{M}$ arbitrarily placed radar nodes and an Access Point (AP) is counted. AP is suited above the circular sensing area.

Each Radar can be a source node that transmits its data or it can be a relay node that supports proceed data of the source node. There are two stages during cooperative communication: (1) radar source node sends data using power P1, and these signals can be overload by AP and the nominated repeat node at the same time. (2) If 
the repeat node decodes the received signals properly, it then resends them with power P2, else it remains quite. Signals received in these two stages can be jointed using Maximum Ratio Combining (MRC) scheme. The signals received by AP are given by the following equations:

$$
\begin{gathered}
y_{s r}(n)=\sqrt{P}_{1} h_{s r}(n) x(n)+\mathrm{n}_{s r}(n) \\
y_{s d}(n)=\sqrt{P}_{1} h_{s d}(n) x(n)+\mathrm{\eta}_{s d}(n) \\
y_{r d}(n)=\sqrt{\widehat{P}_{1}} h_{r d}(n) x(n)+\mathrm{n}_{r d}(n) \\
y(n)=\sqrt{P}_{1} h_{s d} / N_{0} y_{s d}(n)+\sqrt{\widehat{P}_{2}} h_{r d} / N_{0} y_{r d}(n)
\end{gathered}
$$

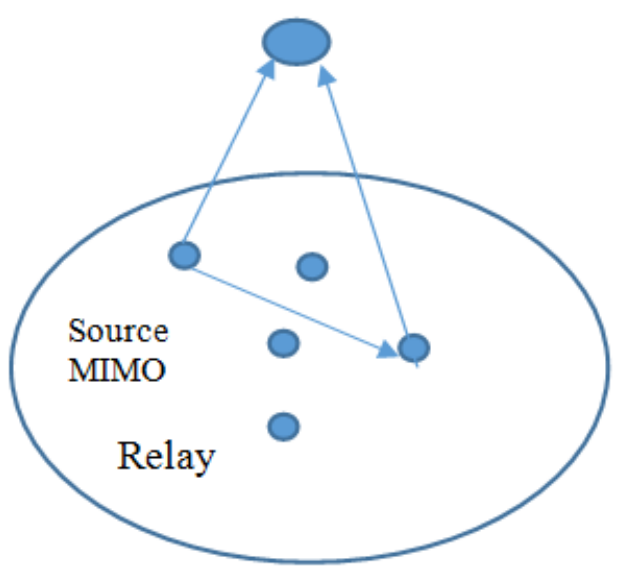

Fig. 1. WSN model with a group of sensor nodes

Where $P_{1}$ is the transmission power of the source node, and $\mathrm{x}(\mathrm{n})$ is the transmitted symbol with unit average power. $h_{s r}(\mathrm{n}), h_{s d}(\mathrm{n})$ and $h_{r d}(\mathrm{n})$ are channel gains of the source-relay, source-BS and relay-BS channels modelled as $\mathrm{CN}\left(0, \sigma_{s r}^{2}\right), \mathrm{CN}\left(0, \sigma_{s d}^{2}\right)$ and $\mathrm{CN}\left(0, \sigma_{r d}^{2}\right)$, respectively.

We assume that instantaneous gains of different links are mutually independent. $\eta_{s d}, \eta_{s r} . \eta_{r d}$ and additive noise modelled as $\mathrm{CN}\left(0, N_{0}\right)$. The repeat node and BS are eligible to decide if the decoded packet is proper by cyclic redundancy check $(\mathrm{CRC})$ code attached to the end of data packet. When the repeat node decodes the received

Packet correctly, $\widehat{P_{2}}=P_{2}$, otherwise, $\widehat{P_{2}}=0$. For slow fading channels, the channel coefficients stay fixed in a time slot and the subscript $\mathrm{n}$ can be omitted. In the following part, optimal power allocation strategy that minimizes the total transmission power expose to BER restriction is obtained at the base station. Suppose that all channel links are obtainable.

$\left(\sigma_{s r}^{2} \neq 0, \quad \sigma_{s d}^{2} \neq 0, \sigma_{r d}^{2} \neq 0\right.$ ), the average BER performance for cooperative communication with M-PSK or MQAM modulation is upper-bounded by [4] :

Let us consider a MIMO radar where $M_{t}, M_{r}$ transmistting and reciveing antennas are colcated. Assume the target located at $(\theta, R)$, the received baseband signal at the $\mathrm{M}$ antenna element of the receiver array, derived from the electric filed mode is given by :

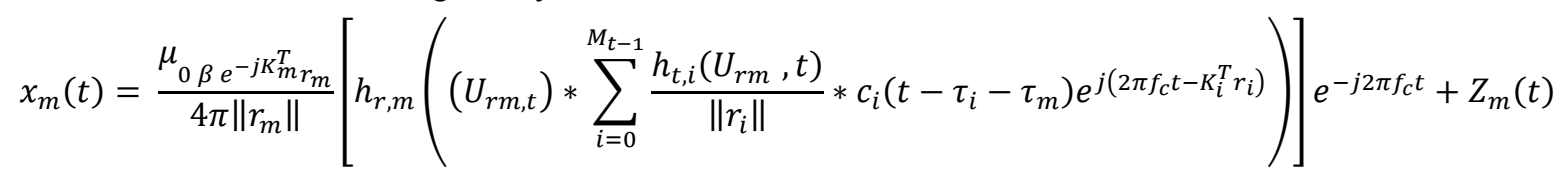

$m=0, \ldots \ldots \ldots, M_{t}-1$ where $\tau_{i}=K_{i}^{T} r_{m} / 2 \pi f_{c}, \quad \tau_{m}=K_{m}^{T} r_{m} / 2 \pi f_{c}$

and $\mathrm{Z}(\mathrm{t})$ is the noise and interfrence recived by $\mathrm{m}^{\text {th }}$ antenna element. Equation (5) can be rewritten by :

$x_{m}(t)=\frac{\mu_{0 \beta} e^{-j K_{m}^{T} r_{m}}}{4 \pi\left\|r_{m}\right\|}\left[h_{r, m}\left(\left(U_{r m, t}\right) * \sum_{i=0}^{M_{t-1}} \frac{h_{t, i}\left(U_{r m}, t\right)}{\left\|r_{i}\right\|} * c_{i}\left(t-\frac{K_{i}^{T} r_{m}}{2 \pi f_{c}}-\frac{K_{m}^{T}}{2 \pi f_{c}}\right) e^{j\left(2 \pi f_{c} t-K_{i}^{T} r_{i}\right)}\right)\right] e^{-j 2 \pi f_{c} t}+$

$Z_{m}(t)(6)$

$$
m=0, \ldots \ldots \ldots, M_{t}-1
$$

where $c_{i}(t)$ is wideband signal, applying freqyency transform to write equation (4) in frequncey doiman we get : 
$x_{m}(t)=\frac{{ }_{0 \beta \beta} e^{-j K_{m}^{T} r_{m}\left(1+\frac{f}{f_{c}}\right)}}{4 \pi\left\|r_{m}\right\|}\left[H_{r, m}\right]\left[\left(\left(U_{r m, f+f_{c}}\right) * \sum_{i=0}^{M_{t-1}} \frac{H_{t, i}\left(U_{r m}, f+f_{c}\right)}{\left\|r_{i}\right\|} e^{-j K_{i}^{T} r}\left(1+\frac{f}{f_{c}}\right)_{i} \quad C_{i}(f)\right)\right]+Z_{m}(f)$

The antenna radiation pattern (7) is given by:

$x_{m}(f)=\alpha \beta \frac{1}{\left\|r_{m}\right\|} e^{-j K_{m}^{T} r_{m}\left(1+\frac{f}{f_{c}}\right)} g_{r, m}\left(\varnothing, f+f_{c}\right) \sum_{i=0}^{M_{t}-1} \frac{1}{\left\|r_{i}\right\|} g_{r, m}\left(\emptyset, f+f_{c}\right) e^{-j K_{i}^{T}} r\left(1+\frac{f}{f_{c}}\right) C_{i}(f)$

Where $X(f)=\left[X_{0}(f) \ldots \ldots X_{M_{r}-1}(f)\right]^{T}, C(f)=\left[C_{0}(f) \ldots \ldots C_{M_{r}-1}(f)\right]^{T}, Z(f)=\left[Z_{0}(f) \ldots \ldots Z_{M_{r}-1}(f)\right]^{T}$ the wideband steering transmitting and receiving vectors are given respectively

$a_{t}(\varnothing, f)=\frac{1}{R} e^{j 2 \pi\left(\frac{f+f_{c}}{v}\right)(D \sin \emptyset+H \cos \emptyset)}\left[g_{t, i}^{*}\left(\varnothing, f, f_{c}\right) e^{j 2 \pi\left(f, f_{c}\right)\left(\frac{M_{t-1}}{2}\right) \frac{d_{t} \sin \emptyset}{v}}\right]_{i=0, \ldots M_{t-1}}$

$a_{r}(\varnothing, f)=\frac{1}{R} e^{j 2 \pi\left(\frac{f+f_{c}}{v}\right)(D \sin \emptyset+H \cos \emptyset)}\left[g_{r, i}^{*}\left(\varnothing, f, f_{c}\right) e^{j 2 \pi\left(f, f_{c}\right)\left(\frac{M_{r-1}}{2}\right) \frac{d_{r} \sin \emptyset}{v}}\right]_{i=0, \ldots M_{r-1}}$

The DOA estimation mechanisms will be completed by two dimension search over parameters $(\emptyset, f)$, in the plane wave case the product $\left(K_{i}^{T} r_{i}\right)$ is given by :

$K_{i}^{T} r_{i}=\frac{2 \pi f_{c}}{v}\left(D-\left(i-\frac{M_{t}-1}{2}\right) d t\right) \sin \emptyset+\frac{2 \pi f_{c}}{v} H \cos \emptyset \quad i=0, \ldots \ldots, M_{t_{-1}}$

After omitting the common to every element of array, the plane wave wideband steering vector will be given by:

$a_{t}\left(\emptyset, f_{c}\right)=\left[g_{t, i}^{*}\left(\varnothing, f_{c}+f\right) e^{j 2 \pi\left(f_{c}+f\right)}\left(\frac{M_{t}-1}{2}-i\right) \frac{d_{t} \sin \emptyset}{v}\right]_{i=0, \ldots \ldots, M_{t}-1}$

$a_{r}\left(\varnothing, f_{c}\right)=\left[g_{r, i}^{*}\left(\varnothing, f_{c}+f\right) e^{j 2 \pi\left(f_{c}+f\right)}\left(\frac{M_{r}-1}{2}-i\right) \frac{d_{r} \sin \varnothing}{v}\right]_{i=0, \ldots \ldots, M_{r}-1}$

Assuming that the radiation patterns of the antenna elements are identical, subsequently, the plane wave steering vectors are finally given as:

$a_{t}\left(\emptyset_{k}, p\right)=\left[e^{j 2 \pi\left(f_{c}+f_{p}\right)\left(\frac{M_{t-1}}{2}-i\right) \frac{d_{t} \sin \phi_{k}}{v}}\right]_{m=0, \ldots, M_{t-1}}$
$a_{r}\left(\emptyset_{k}, p\right)=\left[e^{j 2 \pi\left(f_{c}+f_{p}\right)\left(\frac{M_{r-1}}{2}-i\right) \frac{d_{t} \sin \phi_{k}}{v}}\right]_{m=0, \ldots, M_{t-1}}$

\section{SIMULATION RESULTS}

Figure 2 describes the network lifetime of WSN with Cooperative sensors and cooperative by using DFT. The number of sensors varies from 4 to 14. Obviously, the network lifetime of DFT Cooperative technique is bigger than that of cooperative scheme for any number of sensors. For example, for a WSN with 14 sensors, the DFT cooperative technique enhances the network lifetime by 1.8 times that of cooperative technique.

TABLE I. COMMUNICATION SYSTEM PARAMETERS

\begin{tabular}{|c|c|}
\hline Parameter & Value \\
\hline Carrier Frequency & $1 \mathrm{GHz}$ \\
\hline Packet Size & $512 \mathrm{bits}$ \\
\hline Data Rate & $10 \mathrm{~KB}$ \\
\hline Noise & $-40 \mathrm{~dB}$ \\
\hline$E_{\text {init }}$ & 1 \\
\hline Transmit antenna & 3 \\
\hline Receive antenna & 3 \\
\hline Bit Error Rate (BER) & $10^{-3}$ \\
\hline Number of Targets & 3 \\
\hline Power transmitted & $0.2 \mathrm{~W}$ \\
\hline Raduis of the Network & $100 \mathrm{~m}$ \\
\hline
\end{tabular}




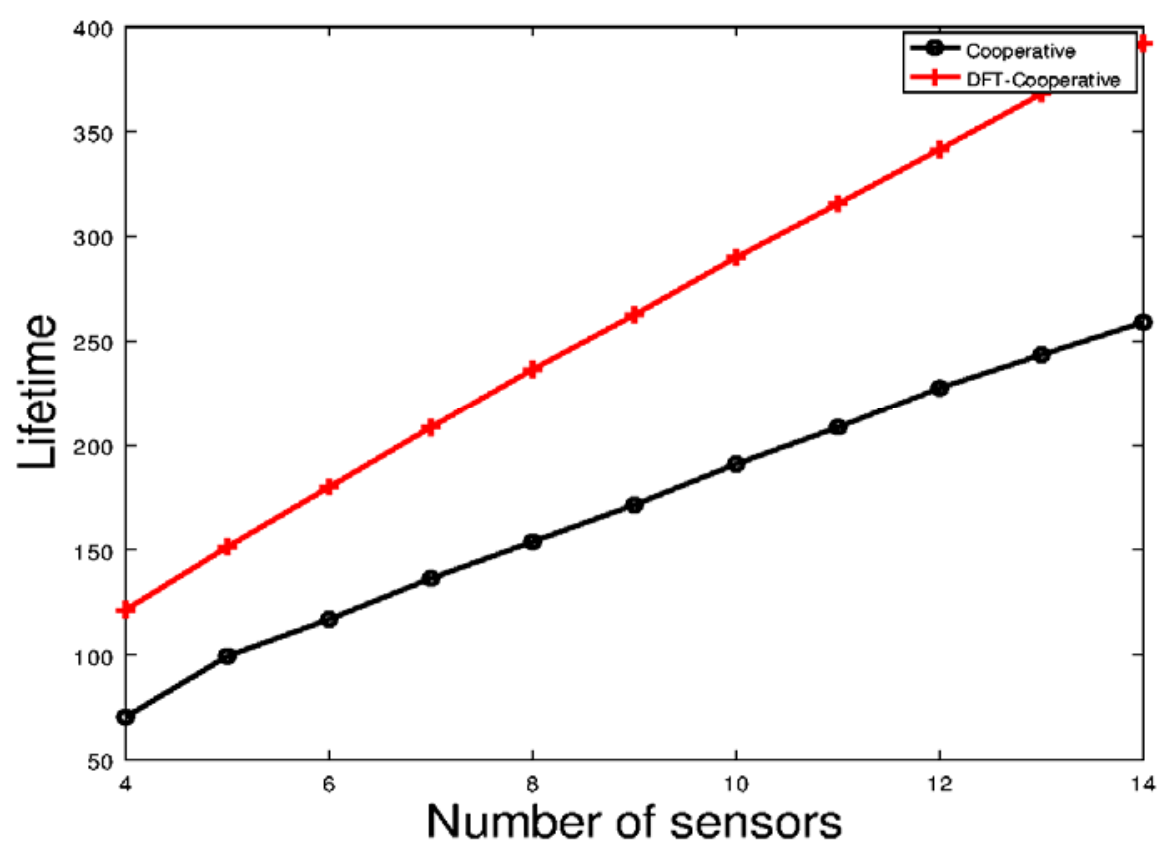

Fig. 2. Comparison of Lifetime for Cooperative and DFT Cooperative cases.

Figure 3 shows the network lifetime of a WSN for both cases: Cooperative and DFT Cooperative under different modulation schemes. We can see from this figure that compared with BPSK, QPSK, and 16PSK modulation, BPSK has much better lifetime performance for both Cooperative and DFT Cooperative, also we can see the DFT Cooperative performs better than Cooperative for all cases of modulations.

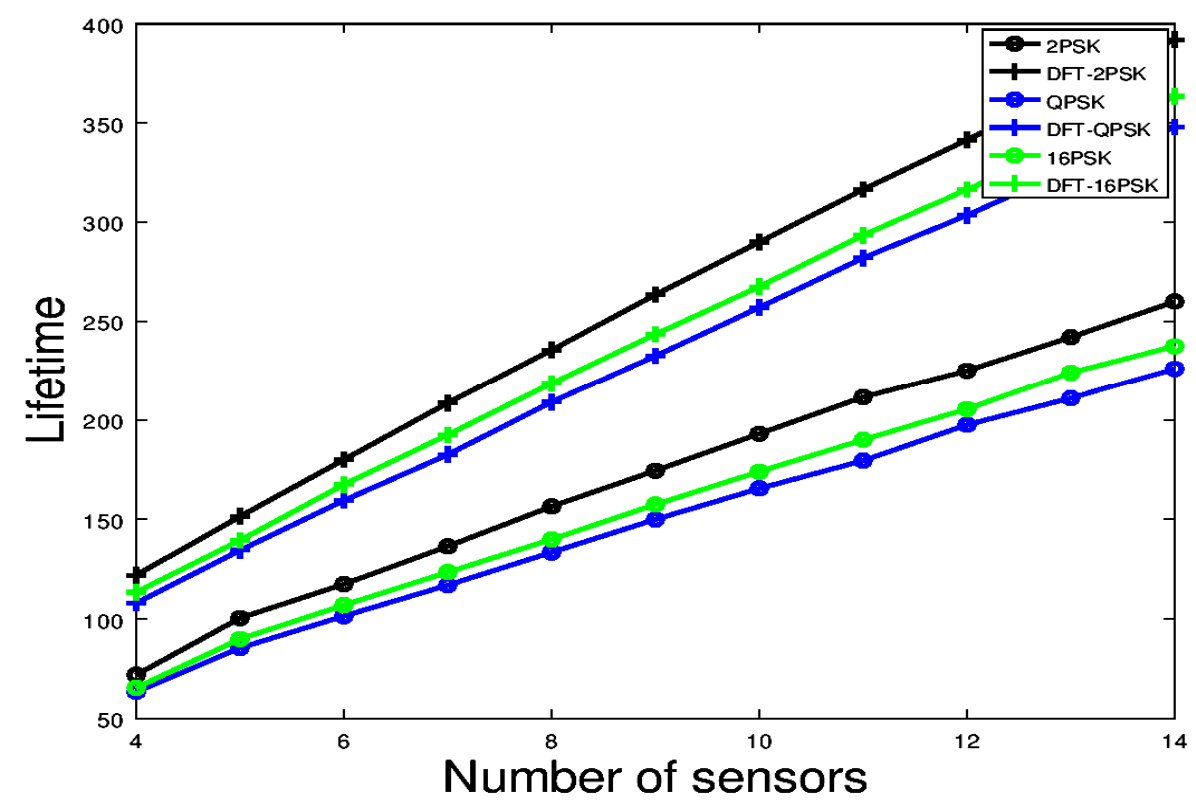

Fig. 3. Lifetime for Cooperative and DFT Cooperative under different Modulation schemes.

Figure 4 depicts the network lifetime over the distinct altitudes of Access Points. The number of sensor nodes is fixed to 100 . We see that DFT Cooperative scheme under different modulations outperforms Cooperative scheme at all heights of access points. In addition, Figure 4 indicates that as the altitude of Access Points increase, the transmission power rises and thus the average network lifetime reduces.

Figure 5 depicts the network lifetime with different BER requirements. We can see from this figure that BPSK performs better than other modulations such as QPSK and 16 PSK. 


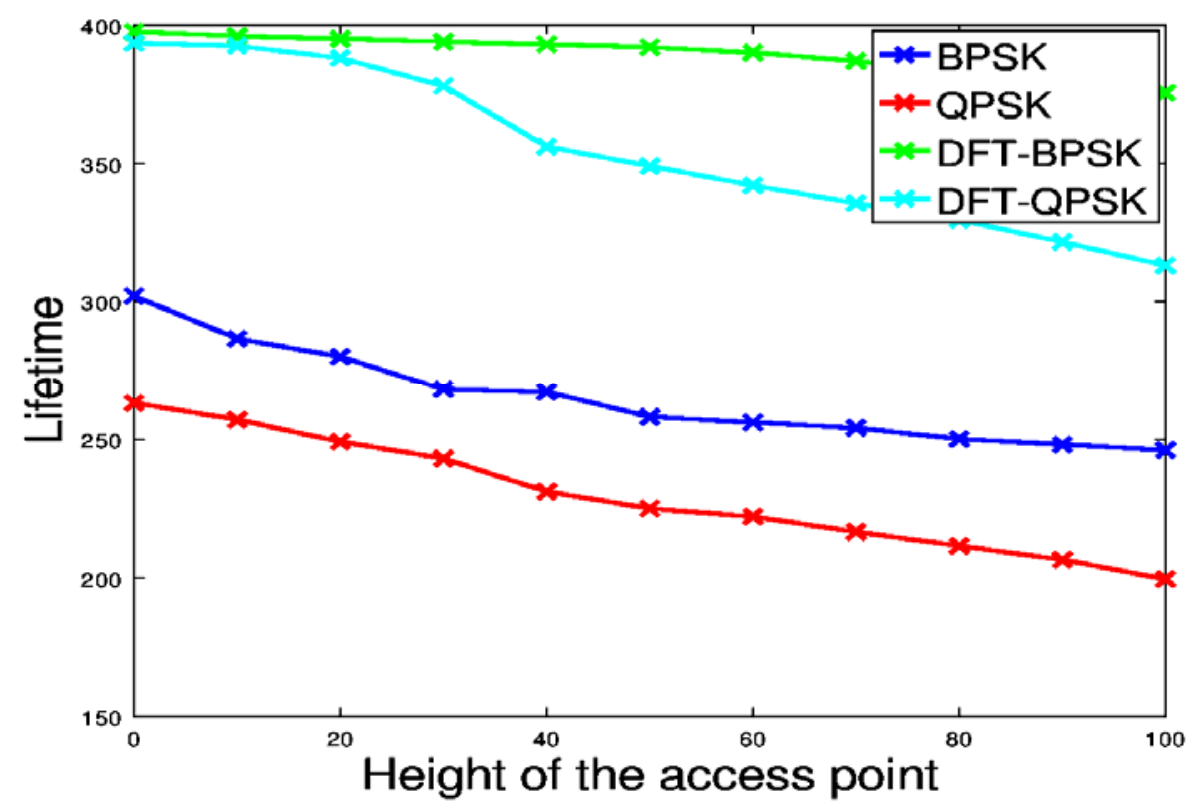

Fig.4. Lifetime for Cooperative and DFT Cooperative over different heights of Access Points.

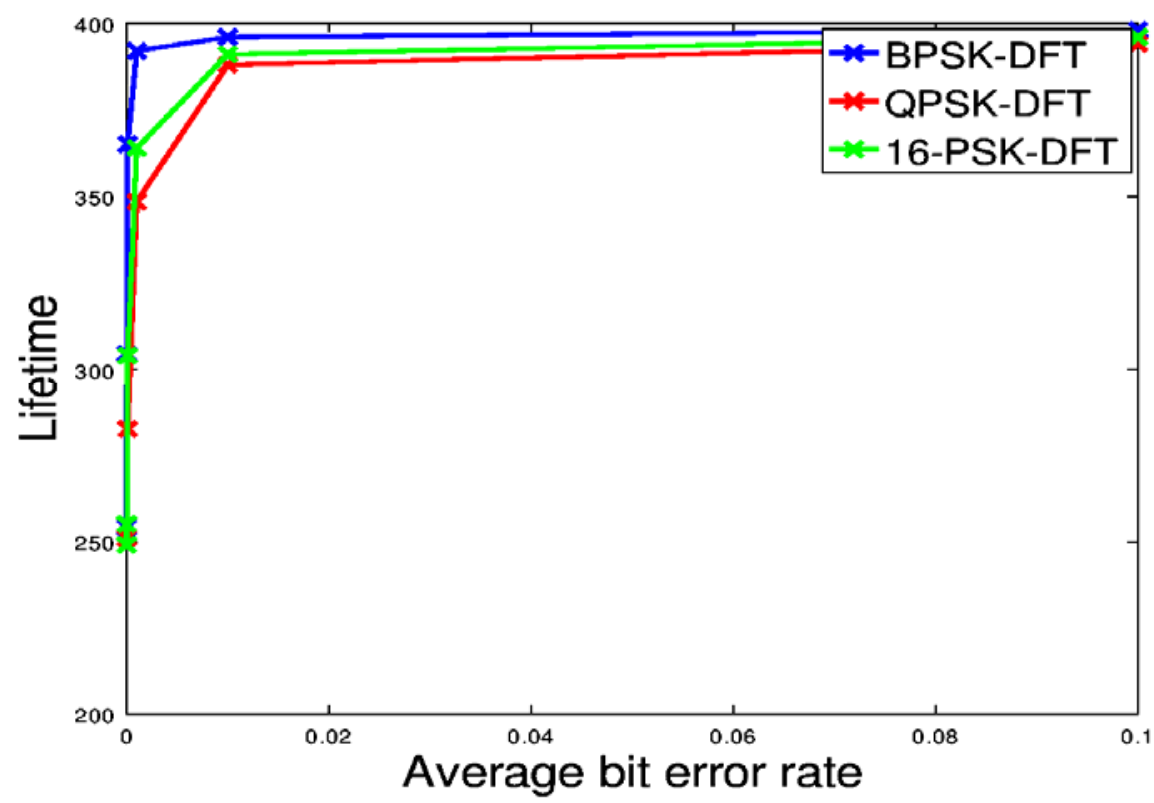

Fig. 5. Lifetime for DFT Cooperative with different BER Constraints.

\section{IV.CONCLUSION}

In this paper, we have considered a new MIMO cooperative based on DFT is proposed to expand the lifetime of wireless sensor networks. The scheme is evaluated to minimize the energy consumption. The simulation results reveal that our protocol improves the network lifetime compared with MIMO cooperative protocol. Also high performance can be obtained when the numbers of sensor nodes are increased. Through simulation results, we also see that with the same average BER constraint the lifetime performance of BPSK is highly superior than QPSK and other modulation scheme.

\section{REFERNCES}

[1] Ouchi, K, "Recent trend and advance of synthetic aperture radar with selected topics," Remote Sens. $2013,5,716-807$.

[2] Wen-Qin. W, Huaizong S, "Radar-to-Radar Interference for Distributed Radar Sensor Networks," Remote sensing, $2014,6,740-755$.

[3] Yunxia Chen, Qing. Z, "Maximizing the Lifetime of Sensor Network Using Local Information on Channel State and Residual Energy," Information Science and Systems Conference, march 2005.

[4] Shuguang. C, Andrea. J, Ahmad. B, "Energy-Efficient of MIMO and Cooperative MIMO Techniques in Sensor Networks," IEEE Journal on Selected Areas in Communications, vol. 22, pp. 1089-1098, Aug. 2004. 
[5] J. N. Laneman, D. Tse, and G. W. Wornell, "Cooperative diversity in Wireless networks: Efficient protocol and outage behavior," IEEE Trans. Inform. Theory. vol. 50, no. 12, pp. 3062-3080, Dec. 2004.

[6] Chao. Z, Ju. L, Lina. Z, and Hongji. X, "Lifetime Maximization Via a New Cooperative MAC Protocol in Wireless Sensor Networks," IEEE GLOBECOM. 2009.

[7] Nuray. AT, and Daraghma S.M, "A New Energy Efficient Clustering-Based Protocol for Heterogeneous Wireless Sensor Networks," Electrical and Electronic Systems, vol. 4, October r 2015.

[8] Nuray. AT, and Raed S. M. Daraghma, "Improved Cluster Head Selection for Energy Efficiency in Wireless Sensor Networks," International Journal of Modern Engineering Research, vol. 6, May 2016.

\section{AUTHOR}

RAED S. M. DARAGHMA was born in 1977 in Palestine, he received his master degree from Jordan science and technology in electrical and communication engineering Jordan in 2010, he got his P.H.D degree from anadolu university, turkey in 2016. He has published number of papers and journals. He has engaged in educational work many years manly teaches digital communication, mobile, digital communication networks at Palestine technical university P.T.U. His mainly research areas include wireless sensor networks, signal processing and MIMO radar. 